\title{
Diagnostic Interstitial Lung Diseases in Patients Having Normal Chest Radiograph through High Resolution Computed Tomography
}

\author{
Aneeq Amoon* \\ Bachelors in Medical Imaging Technology, Department of Allied Health Sciences, Superior College Lahore, \\ University Campus, 17-KM Raiwaind Road, Kot Arain, Lahore, Pakistan
}

Dua Amin

Bachelors in Medical Imaging Technology, Department of Allied Health Sciences, Superior College Lahore, University Campus, 17-KM Raiwaind Road, Kot Arain, Lahore, Pakistan

Muhammad Shahanshah

Bachelors in Medical Imaging Technology, Department of Allied Health Sciences, Superior College Lahore, University Campus, 17-KM Raiwaind Road, Kot Arain, Lahore, Pakistan

Saman Arshad

Bachelors in Medical Imaging Technology, Department of Allied Health Sciences, Superior College Lahore, University Campus, 17-KM Raiwaind Road, Kot Arain, Lahore, Pakistan

Kainat Akbar

Bachelors in Medical Imaging Technology, Department of Allied Health Sciences, Superior College Lahore, University Campus, 17-KM Raiwaind Road, Kot Arain, Lahore, Pakistan

Rana Muhammad Athar Azeem Shams

Masters in Medical Imaging Technology, Lecturer, Department of Allied Health Sciences, Superior College Lahore, University Campus, 17-KM Raiwaind Road, Kot Arain, Lahore, Pakistan

Hafiz Muhammad Rizwan

Masters in Medical Imaging Technology, Lecturer, Department of Allied Health Sciences, Superior College Lahore, University Campus, 17-KM Raiwaind Road, Kot Arain, Lahore, Pakistan

Rana Muhammad Bakhtawar Khan Sajawal

Masters in Mathematics, Lecturer, Department of Allied Health Sciences, Superior College Lahore, University Campus, 17-KM Raiwaind Road, Kot Arain, Lahore, Pakistan

Sybil Rose

Masters in Diagnostic Ultrasound, Lecturer, Department of Allied Health Sciences, Superior College Lahore, University Campus, 17-KM Raiwaind Road, Kot Arain, Lahore, Pakistan

The research is financed by Asian Development Bank. No. 2006-A171(Sponsoring information)

\section{Abstract}

Background: In Pakistan, the main cause of death is interstitial lung disease (ILD) i.e., 4.75\%. In interstitial lung disease majority of the patients is about the age of 57.5 years. The age group maybe varying between the ages of 46 and 65 year. Female gender is more predominantly i.e., $65.6 \%$ in all types of ILD except the interstitial pulmonary fibrosis $(\mathrm{P}<0.001)$.

Objective: The aim of our study is to diagnose interstitial lung diseases in patients having normal chest radiograph through high resolution computed tomography.

Study design: Our study design was cross-sectional descriptive study.

Material and method: The descriptive cross-sectional study was conducted in which data of 100 patients were taken. The data was collected from the Radiology department of Sheikh Zayed Hospital Rahim Yar Khan. After informed consent, data was collected through CT Toshiba 164 slices.

Result: 100 patients were included in our study out of which 61 were females and 39 were male's with the mean age of 49.32 years. The chest radiograph of the patients having ILD shows the patchy ground glass opacities (39.0\%), consolidation of the lungs $(21.0 \%)$, reticular shadowing $(16.0 \%)$ and the pleural effusion $(24.0 \%)$ while on the high-resolution computed tomography the patients were represented with ground glass haze $(42.0 \%)$, calcific foci $(21.0 \%)$, nodular lesion $(16.0 \%)$, consolidation of the lungs $(30.0 \%)$ and pleural effusion $(22.0 \%)$. 
Conclusion: Chest radiograph nearly misses common radiographical features which are suggestive of interstitial lung disease but can be seen on High Resolution Computed Tomography (HRCT) due to its high resolution. So, it can be concluded that the HRCT is more efficient and authentic diagnostic equipment in assessing the Interstitial Lung Disease as compared to the chest radiograph.

Keywords: Interstitial lung disease, Chest radiograph, Computed tomography, High resolution computed tomography.

DOI: $10.7176 / \mathrm{JHMN} / 91-04$

Publication date:July $31^{\text {st }} 2021$

\section{Introduction}

In Pakistan, the main cause of death is interstitial lung disease (ILD) i.e., $4.75 \% .{ }^{-}$ILDs are more common in urban areas as compared to rural areas. The distribution of the sub-types of ILD in rural areas are IPF/UIP is $15.5 \%$, NSIP is $13.0 \%$, HP is $8.6 \%$, CTD-ILD is $5.4 \%$ and sarcoidosis is $5.6 \%$. In urban areas the distributions of sub-types of ILD are IPF/UIP is $84.5 \%$, INSIP is $87.0 \%$, HP is $91.4 \%$, CTD-ILD is $94.6 \%$ and sarcoidosis is $94.4 \%$. The frequency of ILD in different communities are as the IPF is more likely to be in the migrants includes from the regions of Western, Southern and Central Sub-continent is $45.5 \%$, while $21.3 \%$ of Pashtuns, $15.7 \%$ in Punjabis and $6.4 \%$ in Sindhis ${ }^{2}$. In interstitial lung disease majority of the patients is about the age of 57.5 years. The age group maybe varying between the ages of 46 and 65 year. Female gender is more

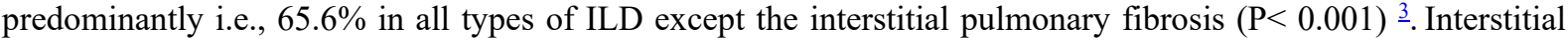
lung disorders are a set of diffuse parenchymal lung problems that are related with incidence and mortality ${ }^{4}$. "Interstitial" can cause misleading as it is related to most of the disorders and it also involves air spaces, peripheral airways and vessels. These disorders can progress to irreversible pulmonary fibrosis $\underline{5}$. The ILD is defined as an intense and persistent two-sided parenchymal infiltrative disease that is associated along with several levels of inflammation and fibrosis $\underline{6}$.

The major categories of ILD are idiopathic interstitial pneumonia (IIP) that are partitioned further into idiopathic aspiratory fibrosis (IPF), non-specific interstitial pneumonia (NSIP), cryptogenic coordinating pneumonia (COP), respiratory bronchiolitis related interstitial lung infection (RB-ILD), desquamative interstitial pneumonia (DIP), intense interstitial pneumonia (AIP), lymphoid interstitial pneumonia (LIP). ILD is likewise connected with connective tissue issue (CTD-ILD), sarcoidosis, hypersensitivity pneumonitis (HP), drug effected ILD and lung infection. Some types of ILD are also correlated along with specific genetic dysfunction e.g., familial pulmonary fibrosis ${ }^{7}$. The prevalence of different types of ILD are as follows; IPF is $34.4 \%$, HP is $17.7 \%$, NSIP is $16.8 \%$, CTD-ILD is $16.3 \%$, sarcoidosis is $9.1 \%$ and $5.7 \%$ are other types of ILDs 2 .

The patients having IPF are presents initially with the signs and symptoms of acute exacerbation, which is categorized by acute worsening of dyspnea and hypoxemia with ground glass opacities $\stackrel{8}{\text {. Other symptoms }}$

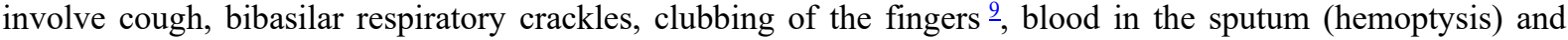
weight loss, patients with these signs and symptoms are suggested for chest radiograph and HRCT, chest radiograph and HRCT are helpful full in the diagnosis of the ILD. HRCT is considered more valid in the detection of the ILD as compared to the chest radiographs $\underline{10}$. Chest radiographs appear abnormal in symptomatic patients. Chest x-ray shows reticular patterns, which is categorized by opacities in the interstitium and minor cystic spaces in the outer layer and is best seen in the costo-diapragmatic recess and in the lower zones of lung 11 . HRCT performs an important character in determination, diagnosis, identification and tracking of the Interstitial

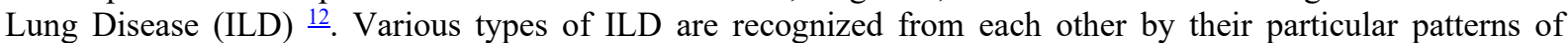
anomalies. Hence HRCT chest scan is helpful in the diagnostic workup of ILD patients $\underline{13}$. HRCT is far better than a radiograph in identifying changes inside the interstitium when chest radiograph is typical $\frac{14}{\text {. The }}$ parameters used in the HRCT scan is collimation of $1 \mathrm{~mm}$, intervals of $10 \mathrm{~mm}$ and $1 \mathrm{~mm}$ thickness of high spatial resolution. $\underline{15}$

The aim of our research provides the descriptive statistic regarding the patients who have clinical manifestations of Interstitial Lung Disease (ILD). Chest radiograph may be normal initially and show not many anomalies thus incapable to distinguish the particular diagnosis of the ILD. High Resolution Computed Tomography of lungs in the event of doubted ILD is an important device for precise and early distinguishing proof for assessment of lung parenchyma. HRCT surveys the presence of the infection in the lungs, sort of the sickness and changes of dynamic lungs illness.

\subsection{Material and Methods}

The descriptive cross-sectional study was conducted in which data of 100 patients were taken. The data was collected from the Radiology department of Sheikh Zayed Hospital Rahim Yar Khan. After informed consent, data was collected through CT Toshiba 164 slices. Out of 100 patients 61 were females and 39 were males. The minimum age observed was 15 years and the maximum age observed was 87 years. The mean age involved was 49.32 years. Study duration was of 4 months. Inclusion criteria includes was patients with dry cough, Asthma, 
Dyspnea, weight loss, clubbing, hemoptysis (blood with sputum), fever and unremarkable chest x-ray. Exclusion criteria includes was patients with non-respiratory related diseases, lung cancer and metastasis are not observed in our study.

\section{Results}

100 patients were included in our study out of which 61 were females and 39 were males. The minimum age was 15 years and the maximum age was 87 years with the mean age of 49.32 years. The chest radiograph of the patients having ILD shows the patchy ground glass opacities $39.0 \%$, consolidation of the lungs $21.0 \%$, reticular shadowing $16.0 \%$ and pleural effusion $24.0 \%$ while on the High-resolution computed tomography the patients were represented with ground glass haze $42.0 \%$, calcific foci $21.0 \%$, nodular lesion $16.0 \%$, consolidation of the lungs $30.0 \%$ and pleural effusion $22.0 \%$.

\section{Discussion}

It was a cross-sectional study in which 100 patients of Interstitial Lung diseases are involved. Out of which 61 were females and 39 were males. Nadiah. M et al. in 2009 conducted a study to determine the characteristics of Rheumatoid Arthritis patients with lung disease in relation to clinical characteristics, pulmonary function tests (PFTs) and HRCT thorax. Their study concluded that there was no association between the male gender, smoking, rheumatoid factor, extra-articular manifestations, activity, severity and duration of the disease ${ }^{16}$.

The mean age involved in our study was 49.32 years. The minimum age observed was 15 years and the maximum age observed was 87 years. Ya-Song Li et al. 2010 conducted a study to describe the clinical significance of HRCT in evaluation of patients with rheumatoid arthritis associated ILD. 79 females and 31 males were diagnosed with rheumatoid arthritis. The study concluded that the old age, smoking and pulmonary rales were closely related to $\mathrm{ILD}^{17}$. There was another study conducted by Sonila Dauti et al. in 2018 that was aimed to determine the independent risk factors that was involved in the development of interstitial lung disease and the study concluded that Smoking, hepatitis C, history of tuberculosis, history of pneumonia, chronic obstructive pulmonary disease, male sex, and older age were significantly associated with interstitial lung disease development $\underline{18}$. Mariana Lima et al. 2019 performed a study to describe the clinical, functional, and radiological features of index cases of familial pulmonary fibrosis (FPF) and concluded that the most HRCT scans of these patients' exhibit patterns not typical of usual interstitial pneumonia. The family history of fibrotic lung diseases should be investigated in all patients under suspicion, regardless of their age $\underline{19}$.

In our study the HRCT scans shows the appearances ground glass haze in $42.0 \%$, calcific foci in $21.0 \%$, nodular lesions in $16.0 \%$, consolidation of the lungs in $30.0 \%$ and pleural effusion in $22.0 \%$. M. Ali Karimi et al. 2014 conducted a study to portray the range of radiological findings on high resolution computed tomography (HRCT) scans in patients with Cryptogenic Organizing Pneumonia (COP) and their study concluded that the fundamental introductions of COP on HRCT incorporate reciprocal GGOs and consolidations in the lower projections of the lung $\underset{20}{ }$. Igor Murad Faria et al. 2015 conducted a study on 36 patients of organizing pneumonia out of which 26 were females and 10 were males. Their study concluded that ground-glass opacities and consolidation were the most well-known discoveries, with a dominatingly arbitrary dispersal, despite the fact that they were more normal in the center and lower thirds of the lungs $\underline{21}$. Mohit Sharma et al. 2020 conducted a cross-sectional study to assess the HRCT findings in patients with rheumatoid arthritis associated lung diseases (RA-ILD). On HRCT, reticulations were present in $35.7 \%$ of patients: honey combing was present in $53.6 \%$ of patients, ground glass opacities in $42.9 \%$, pleural thickening in $10.7 \%$, pleural effusion in $7.1 \%$, and pulmonary vascular prominence in $7.1 \%$, rheumatoid nodules in $7.1 \%$ and bronchiectasis in $7.1 \%$. Their study concluded that in the present study, UIP is the most common ILD. Honey combing and ground glass opacities most common finding on HRCT in these patients $\underline{22}$.

The final diagnosis of our study shows that there were further types of Interstitial lung disease such as chronic inflammatory pulmonary disease, interstitial pneumonitis, chronic inflammatory changes , pleural effusion, post inflammatory changes, right lung abscess, scleroderma and COVID-19. Jian Cai et al. 2020 conducted the aim of their study was to compare chest HRCT lung signs identified in scans of differently aged patients with COVID-19 infections and their study concluded that chest HRCT manifestations in patients with COVID-19 are related to patient's age, and HRCT signs may be milder in younger patients ${ }^{23}$. Ananya Mondal et al. conducted a study and concluded that the idiopathic pulmonary fibrosis was the most common type of ILD and the HRCT was the modality of choice in detecting the diffuse parenchymal lung disease especially in the activity, detection and the depiction of the disease ${ }^{24}$.

Our study concluded that the HRCT was more efficient diagnostic equipment in assessing of the interstitial lung disease as compared to the chest radiograph. A study presented by Ahmed Mohamed Osman et al. in 2017 concluded that the HRCT of the chest has a reasonable role in the diagnosis of interstitial lung diseases and has the ability to be classify according to the pattern of involvement, distribution and extent of the disease as well as to estimate the severity and help in prognosis of the disease process $\stackrel{25}{ }$. S. Annapurna et al. conducted a cross 
sectional study. The aim of their study was to determine the radiological profile in a clinically suspected cases of Interstitial Lung Disease. In their study out of 50 clinically suspected patients of interstitial lung disease, a specific pattern was observed in 44 patients on HRCT and 33 patients on chest radiograph. Patterns found on chest radiograph were correlated with patterns found on HRCT. Out of 44 interstitial lung diseases on HRCT, 28 patients revealed correlation between chest radiograph and HRCT pattern. Out of 50, thirty-one patients had correlation between chest radiograph and HRCT findings. Their study concluded that the HRCT is superior to chest radiograph in detection of all basic patterns associated with ILD and scores more in their distribution and lung parenchymal involvement $\frac{26}{}$. Ayesha Tahir et al. conducted a similar study with 148 patients of ILD and concluded that the HRCT was more accurate and highly reliable technique or diagnostic tool to diagnose the ILD as compared to the chest X-ray. The findings of our study were similar with all the above studies we took the patients with signs and symptoms of ILD having normal chest radiograph and the HRCT was performed to detect the presence of ILD in the patients ${ }^{27}$.

According to our study HRCT was the most efficient tool in detecting the Interstitial lung disease because the ground-glass haze appearance, consolidation of the lungs, calcific foci, pleural effusion and the nodular lesions were found more efficient on HRCT and also helpful in the detection of other types of lung diseases that are associated with the interstitial lung disease.

\section{Conclusion}

Chest radiograph nearly misses common radiographical features which are suggestive of interstitial lung disease but can be seen on High Resolution Computed Tomography (HRCT) due to its high resolution. So, it can be concluded that the HRCT is more efficient and authentic diagnostic equipment in assessing the Interstitial Lung Disease as compared to the chest radiograph.

\section{References}

1. Jafri S, Ahmed N, Saifullah N, Musheer M. Epidemiology and Clinico-radiological features of Interstitial Lung Diseases. Pakistan journal of medical sciences 2020;36(3):365.

2. Zubairi ABS, Ansarie M, Mehmud T, Ashraf S, Rao NA, Saeed S, et al. Disease.

3. Zubairi A, Ansarie M. NATIONAL REGISTRY OF INTERSTITIAL LUNG DISEASE FROM PAKISTAN. Chest 2020;158(4):A2616.

4. Antoniou KM, Margaritopoulos GA, Tomassetti S, Bonella F, Costabel U, Poletti V. Interstitial lung disease. European Respiratory Review 2014;23(131):40-54.

5. Ward J, McDonald C. Interstitial lung disease: An approach to diagnosis and management. Australian family physician 2010;39(3):106.

6. Meyer KC, Raghu G, Baughman RP, Brown KK, Costabel U, du Bois RM, et al. An official American Thoracic Society clinical practice guideline: the clinical utility of bronchoalveolar lavage cellular analysis in interstitial lung disease. American journal of respiratory and critical care medicine 2012;185(9):1004-14.

7. Meyer KC. Diagnosis and management of interstitial lung disease. Translational respiratory medicine 2014;2(1):1-13.

8. Thomson CC, Duggal A, Bice T, Lederer DJ, Wilson KC, Raghu G. 2018 Clinical practice guideline summary for clinicians: diagnosis of idiopathic pulmonary fibrosis. Annals of the American Thoracic Society 2019;16(3):285-90.

9. Raghu G, Collard HR, Egan JJ, Martinez FJ, Behr J, Brown KK, et al. An official ATS/ERS/JRS/ALAT statement: idiopathic pulmonary fibrosis: evidence-based guidelines for diagnosis and management. American journal of respiratory and critical care medicine 2011;183(6):788-824.

10. Lynch JP, Weigt SS, Suh RD. Thoracic imaging for diffuse ILD and bronchiolar disorders. In: Interstitial Pulmonary and Bronchiolar Disorders: CRC Press; 2016. p. 47-76.

11. Spagnolo P, Sverzellati N, Rossi G, Cavazza A, Tzouvelekis A, Crestani B, et al. Idiopathic pulmonary fibrosis: an update. Annals of medicine 2015;47(1):15-27.

12. Oikonomou A. Role of imaging in the diagnosis of diffuse and interstitial lung diseases. Current opinion in pulmonary medicine 2014;20(5):517-24.

13. Patel T, Ilangovan G, Balganesan H. Comparative Study of X-ray and High Resolution Computed Tomography in the Diagnosis of Interstitial Lung Disease.

14. Baskey S, Mohan C, Toppo SK. High Resolution Computed Tomographic Evaluation Of Patients Suspected Of Having Diffuse Interstitial Lung Diseases With Radiographic Correlation.

15. Man MA, Dantes E, Domokos Hancu B, Bondor CI, Ruscovan A, Parau A, et al. Correlation between transthoracic lung ultrasound score and HRCT features in patients with interstitial lung diseases. Journal of clinical medicine 2019;8(8):1199.

16. MOHD NOOR N, MOHD SHAHRIR MS, Shahid MS, ABDUL MANAP R, SHAHIZON AZURA AM, AZHAR SHAH S. Clinical and high resolution computed tomography characteristics of patients with 
rheumatoid arthritis lung disease. International journal of rheumatic diseases 2009;12(2):136-44.

17. Zou Y-Q, Li Y-S, Ding X-N, Ying Z-H. The clinical significance of HRCT in evaluation of patients with rheumatoid arthritis-associated interstitial lung disease: a report from China. Rheumatology international 2012;32(3):669-73.

18. Choi W-I, Dauti S, Kim HJ, Park SH, Park JS, Lee CW. Risk factors for interstitial lung disease: a 9-year Nationwide population-based study. BMC pulmonary medicine 2018;18(1):1-7.

19. Hortense AB, Santos MKd, Wada D, Fabro AT, Lima M, Rodrigues S, et al. Familial pulmonary fibrosis: a heterogeneous spectrum of presentations. Jornal Brasileiro de Pneumologia 2019;45(5).

20. Mehrian P, Shahnazi M, Dahaj AA, Bizhanzadeh S, Karimi MA. The spectrum of presentations of cryptogenic organizing pneumonia in high resolution computed tomography. Polish journal of radiology 2014;79:456.

21. Faria IM, Zanetti G, Barreto MM, Rodrigues RS, Araujo-Neto CA, Escuissato DL, et al. Organizing pneumonia: chest HRCT findings. Jornal Brasileiro de Pneumologia 2015;41(3):231-7.

22. Wangnoo A, Banotra P, Sharma M, Mahajan A. Author Desk.

23. Chen Z, Fan H, Cai J, Li Y, Wu B, Hou Y, et al. High-resolution computed tomography manifestations of COVID-19 infections in patients of different ages. European journal of radiology 2020;126:108972.

24. Ghosh MK, Sur P, Rahaman M, Ghosh SK, Sau R, Haldar R, et al. High Resolution Computed Tomography Scan Assessment of Different Interstitial Lung Diseases.

25. Hanna MMF, Mohamed AM, Osman AM. Role of high resolution computed tomography in diagnosis of interstitial lung diseases in patients with collagen diseases. The Egyptian Journal of Hospital Medicine 2017;68(2):1135-40.

26. Annapurna S, Badarke P, Chandra E. Study of Interstitial Lung Disease with reference to radiological profile. Asian Journal of Medical Radiological Research 2018;6(1):15-21.

27. Tahir A, Hafeez H, Afzal N, Malik SS, Farooq MY, Saleem N, et al. Findings of High Resolution Computed Tomography in Patients Presenting with Signs and Symptoms of Interstitial Lung Disease Having Normal Chest X-Ray. Journal of Health and Medical Sciences 2019;2(3).

\section{Notes}

Note 1. This is an example.

Note 2. This is an example for note 2

\begin{tabular}{|c|c|c|c|c|c|c|c|c|c|c|c|}
\hline \multicolumn{12}{|c|}{ Statistics } \\
\hline & & Gender & $\begin{array}{c}\text { X-Ray } \\
\text { Findings }\end{array}$ & $\begin{array}{l}\text { Ground } \\
\text { glass } \\
\text { haze } \\
\text { for CT }\end{array}$ & $\begin{array}{l}\text { Calcific } \\
\text { Foci CT } \\
\text { Findings }\end{array}$ & $\begin{array}{l}\text { Nodular } \\
\text { Lesion } \\
\text { CT } \\
\text { Findings }\end{array}$ & $\begin{array}{c}\text { Age } \\
\text { (Years) }\end{array}$ & $\begin{array}{l}\text { Consolidation } \\
\text { CT Findings }\end{array}$ & $\begin{array}{c}\text { Pleural } \\
\text { Effusion } \\
\text { CT } \\
\text { Findings }\end{array}$ & $\begin{array}{c}\text { Other } \\
\text { Findings }\end{array}$ & $\begin{array}{l}\text { CT Final } \\
\text { Diagnosis }\end{array}$ \\
\hline \multirow[t]{2}{*}{$\bar{N}$} & Valid & 100 & 100 & 100 & 100 & 100 & 100 & 100 & 100 & 100 & 100 \\
\hline & Missing & 0 & 0 & 0 & 0 & 0 & 0 & 0 & 0 & 0 & 0 \\
\hline \multicolumn{2}{|l|}{ Mean } & .39 & 2.25 & .42 & .21 & .16 & 49.32 & .30 & .22 & .49 & 2.73 \\
\hline \multicolumn{2}{|l|}{ Median } & .00 & 2.00 & .00 & .00 & .00 & 50.00 & .00 & .00 & .00 & 2.00 \\
\hline \multicolumn{2}{|c|}{ Std. Deviation } & .490 & 1.209 & .496 & .409 & .368 & 18.914 & .461 & .416 & .502 & 2.164 \\
\hline \multicolumn{2}{|l|}{ Minimum } & 0 & 1 & 0 & 0 & 0 & 15 & 0 & 0 & 0 & 1 \\
\hline \multicolumn{2}{|l|}{ Maximum } & 1 & 4 & 1 & 1 & 1 & 87 & 1 & 1 & 1 & 10 \\
\hline \multirow[t]{3}{*}{ Percentiles } & 25 & .00 & 1.00 & .00 & .00 & .00 & 33.00 & .00 & .00 & .00 & 1.00 \\
\hline & 50 & .00 & 2.00 & .00 & .00 & .00 & 50.00 & .00 & .00 & .00 & 2.00 \\
\hline & 75 & 1.00 & 3.00 & 1.00 & .00 & .00 & 62.00 & 1.00 & .00 & 1.00 & 4.00 \\
\hline
\end{tabular}

Table 1. Statistical analysis of X-ray and High-resolution CT Findings 


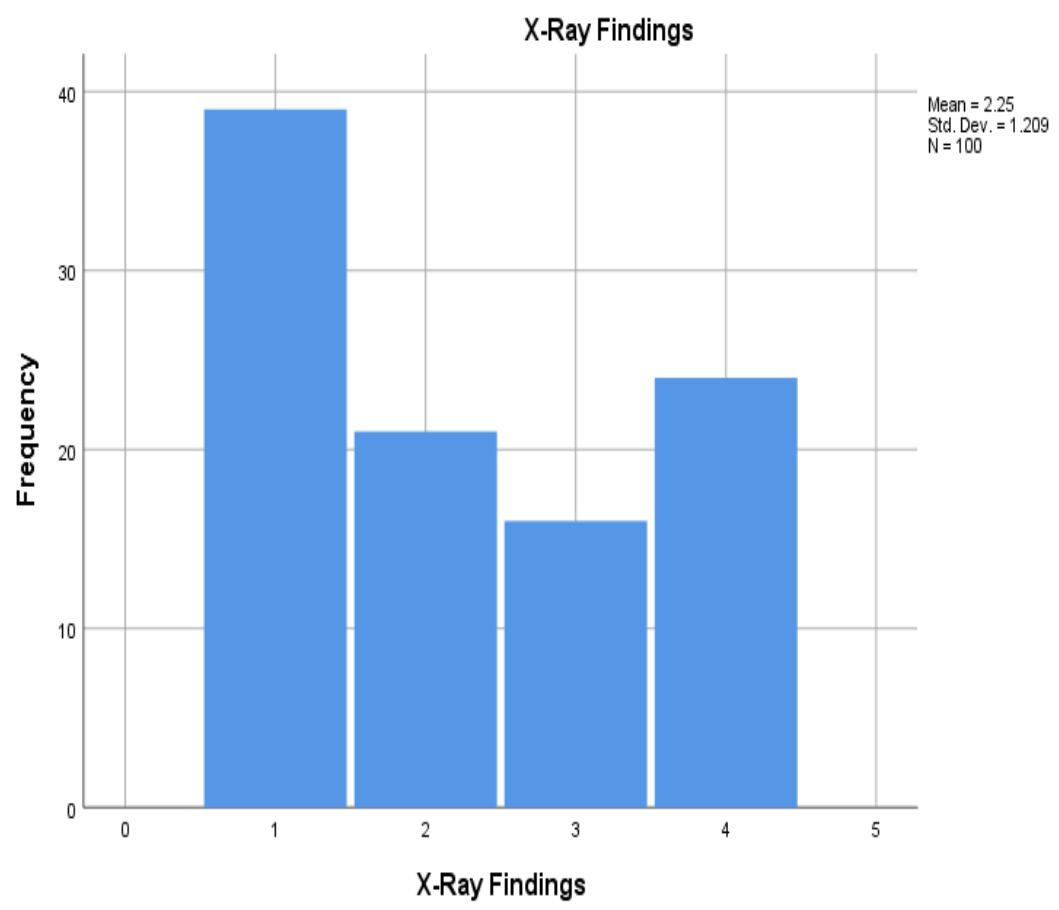

Figure 1. The frequency of the CT findings.

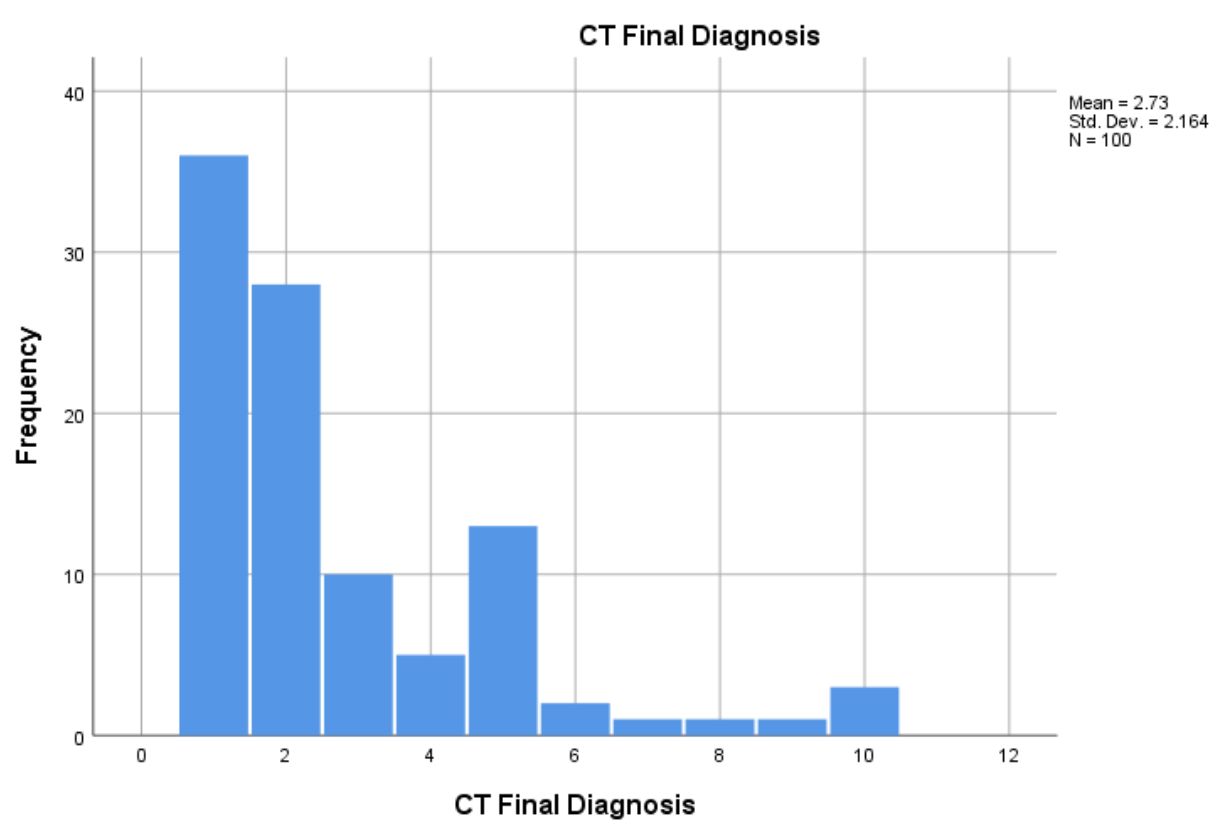

Figure 2. The Statistical values of the gender, x-ray findings, ground glass haze, calcific foci, nodular lesions, age, consolidation of the lungs, pleural effusion on the CT, other findings and the diagnosis on CT 